\title{
Das nordische Modell unter Anpassungsdruck
}

\author{
Claudia Bogedan, Uwe Optenhögel, Hartmut Seifert
}

Der Mythos ist alt. Schon zu Zeiten von Willy Brandt, Olof Palme und Bruno Kreisky - als auch das Modell Deutschland noch ein Erfolg war - hatte das skandinavische Wirtschafts- und Gesellschaftsmodell international einen legendär guten Ruf. Vielen Sozialdemokraten und Linken galt der Norden als Vorbild und Referenz für gesellschafts- und wirtschaftspolitische Konzepte.

$\mathrm{Zu}$ Beginn der 1990er Jahre erlebten die nordischen Länder ihre schwerste Wirtschaftskrise seit der großen Depression Ende der 1920 er Jahre. Umso erstaunter war man, als sich zum Ende der Dekade abzeichnete, dass ausgerechnet sie die Herausforderungen der neuen Zeit besser gemeistert hatten als die übrigen entwickelten Industrieländer.

Der Mythos der nordischen Länder kehrte zurück. Das hat gute Gründe. Nahezu sämtliche Benchmarkingvergleiche und Rankinglisten platzieren die nordischen Länder auf Spitzenplätzen, sei es bei den Nachhaltigkeitsdaten der OECD, bei der PISA-Studie oder bei den Reports des Weltwirtschaftsforums über die globale Konkurrenzfähigkeit. In der wissenschaftlichen Debatte über die Sozial- und Wirtschaftsmodelle in der Welt rangiert das nordische Modell unter dem Gesichtspunkt der Effizienz und Effektivität deutlich vor den kontinentaleuropäischen Ländern. Das Wirtschaftswachstum in Skandinavien lag in den vergangenen Jahren über dem EU-Durchschnitt. Die Beschäftigungsquoten übertreffen bereits seit Jahren die von der EU angestrebten Zielmarken; das gilt im Besonderen für Frauen und ältere Beschäftigte. Die Arbeitslosigkeit liegt unter dem EU-Durchschnitt.

Insgesamt hat sich das nordische Modell als Gegenentwurf zum lange einseitig favorisierten angelsächsischen Modell bewährt. Es widerlegt die lange Zeit verbreitete Auffassung, dass sich eine gute Performanz der Wirtschaft und des Arbeitsmarktes einerseits und eine hohe Staatsquote sowie umfassende Sozialleistungen andererseits ausschließen. Auffallend ist auch, dass die nordischen Länder im internationalen Vergleich noch immer durch eine hohe soziale Gleichheit und ein hohes Lohnniveau hervorstechen. Die skandinavischen Volkswirtschaften belegen, dass Prosperität und Gleichheit sowie Flexibilität und Sicherheit nicht im Widerspruch zueinander stehen.

Seit geraumer Zeit blicken deshalb sowohl die sozialwissenschaftliche Forschung als auch die praktische Politik verstärkt nach Norden, so auch diese Ausgabe der WSI-Mitteilungen. Dabei wird Skandinavien häufig als eine politische und kulturelle Einheit betrachtet. Beim näheren Hinsehen zeigen sich jedoch nicht unbeträchtliche Unterschiede. Das gilt nicht nur für die Konstruktion der nationalen Wirtschafts- und Sozialmodelle, sondern auch für die Muster der Krisenbewältigung.

Die Beiträge zeigen, dass die Anpassungsstrategien in der Wirtschafts-, Arbeitmarkt- und Sozialpolitik sowie im korporatistischen Miteinander von Staat und Sozialpartnern durchaus unterschiedlich waren. Die Beiträge von Thomas Bredgaard et al., Mikkel Mailand, Jon Erik Dølvik und Sven Jochem beschreiben und erläutern, wie die nordischen Arbeitsmärkte sowie die Arbeitsmarkt- und Beschäftigungspolitik reformiert wurden. Wie haben sich die Länder des Nordens an die veränderten Bedingungen von globalem Wettbewerb und neuen Formen des Arbeitens angepasst? Welchen Wandel hat das nordische Modell selbst angesichts der durchgeführten Reformen erfahren?

Beeinflusst wurde diese Transformation seit Beginn des neuen Jahrhunderts durch veränderte politische Machtkonstellationen zunächst in Dänemark, später dann auch in Schweden und Finnland. Die konservativen Regierungen bauen - wenn auch sanft - das System um. Die dabei deutlich werdenden unterschiedlichen Reformstrategien zeigen, dass das bislang so erfolgreiche Modell sich wandeln und in Zukunft weiter ausdifferenzieren wird.

Das wegen seiner offensichtlichen Erfolge bewunderte nordische Modell durchläuft eine Phase der Neuausrichtung. Der konservative schwedische Finanzminister Anders Borg bezeichnete diesen Prozess im Oktober 2008 bei der von ihm gehaltenen Ludwig-Erhard-Lecture der "Initiative Neue Soziale Marktwirtschaft“ als Neuerfindung des schwedischen Modells. Genau darum geht die politische Auseinandersetzung in den nordischen Ländern: Borgs Formulierung lässt sich zwar als Absage an einen Modellwechsel interpretieren. Sie dokumentiert so den konservativen Versuch, die Erfolgsmarke „nordisches Modell" beizubehalten, sie aber mit eigenem politischen Inhalt $z u$ füllen. Das vorliegende Schwerpunktheft greift einige Aspekte der Debatte um die Neuausrichtung auf. Beiträge von Urban Lundberg, Lena Schröder und Joakim Palme et al. vermitteln uns, dass auch im Norden nicht alles eitel Sonnenschein ist; einige Schatten fallen auf das in der deutschen Öffentlichkeit häufig allzu positive Bild von Skandinavien. In diesem Heft kommen daher (fast) ausschließlich skandinavische Autorinnen und Autoren zu Wort, die uns ihre Erfahrungen mit dem nordischen Modell vermitteln. Die Frage, welche Schlussfolgerungen für die Reformdebatte in Deutschland gezogen werden können, ist von anderen zu beantworten. Unser besonderer Dank gilt dem Auslandsbüro der Friedrich-Ebert-Stiftung in Stockholm für seine Unterstützung.

\footnotetext{
Konzept und Koordination des Schwerpunktheftes: Claudia Bogedan, Wissenschaftlerin im WSI in der Hans-BöcklerStiftung. Arbeitsschwerpunkte: Sozial- und Arbeitsmarktpolitik, Skandinavien. e-mail: claudia-bogedan@boeckler.de Dr. Uwe Optenhögel, Leiter des Nordischen Büros der Friedrich-EbertStiftung, Stockholm. Arbeitsschwerpunkte: Arbeitsbeziehungen, Außen- und Sicherheitspolitik. e-mail: uwe.optenhoege/@fesnord.se Dr. Hartmut Seifert, Leiter der Abteilung WSI in der Hans-BöcklerStiftung. Arbeitsschwerpunkte: Arbeitszeit- und Arbeitsmarktforschung. e-mail: hartmut-seifert@boeckler.de
} 